\title{
ON THE CONVERGENCE OF BOUNDED SOLUTIONS OF NON HOMOGENEOUS GRADIENT-LIKE SYSTEMS
}

\author{
Minh-Phuong TRAN ${ }^{1, *}$, Thanh-Nhan NGUYEN ${ }^{2}$ \\ ${ }^{1}$ Faculty of Mathematics - Statistics, Ton Duc Thang University, Ho Chi Minh City, Vietnam \\ ${ }^{2}$ Department of Mathematics, HCMC University of Education, \\ Quarter 6, Linh Trung Ward, Thu Duc District, Ho Chi Minh City, Vietnam \\ *tranminhphuong@tdt.edu.vn \\ (Received: 13-February-2017; accepted: 28-April-2017; published: 8-June-2017)
}

DOI: http://dx.doi.org/10.25073/jaec.201711.50

\begin{abstract}
We study the long time behavior of the bounded solutions of non homogeneous gradient-like system which admits a strict Lyapunov function. More precisely, we show that any bounded solution of the gradient-like system converges to an accumulation point as time goes to infinity under some mild hypotheses. As in homogeneous case, the key assumptions for this system are also the angle condition and the Kurdyka-Lojasiewicz inequality. The convergence result will be proved under a $L^{1}$-condition of the perturbation term. Moreover, if the Lyapunov function satisfies a Lojasiewicz inequality then the rate of convergence will be even obtained.
\end{abstract}

\section{Keywords}

Asymptotic behavior; Gradient-like system; Kurdyka-Lojasiewicz inequality.

\section{Introduction}

We are interested in the long time behavior of bounded solutions of the first order non homogeneous gradient-like system

$$
u^{\prime}(t)+G(t)=f(t), t \geqslant 0
$$

where $u \in C^{1}\left(\mathbb{R}^{+}, \mathbb{R}^{N}\right), G \in C\left(\mathbb{R}^{N}, \mathbb{R}^{N}\right)$, and $f \in L^{1}\left(\mathbb{R}^{+}, \mathbb{R}^{N}\right)$. The second term $f(t)$ in the right hand side of (1) can be interpreted as a perturbation term for the original equation

$$
u^{\prime}(t)+G(u)=0
$$

Roughly speaking, we study in this paper the effect of adding a $L^{1}$ forcing term to the equation (2) on the long time behavior of the trajectories $u$. As in some existing papers on convergence for gradient-like system (2) (see [6] or [15]), we also restrict our study to situations that the system (1) admits a strict Lyapunov function $F$. That means $F(u(t))$ is non increasing and the solution $u(t)$ will be constant if $F(u(t))$ vanishes at some $t$.

The most simple situation of $(2)$ is the case of gradient system where $G=\nabla F$. This system has been studied by many authors such as Absil \& Kurdyka [1, Chill [5], Haraux \& Jendoubi [11], 12] or Simon [17. They have proved that if $F$ satisfies a Lojasiewicz inequality then the bounded solution converges to an equilibrium as $t$ goes to infinity. More general, in a paper of R. Chill et al. [6], the authors gave an abstract result which guarantees that the convergence result also holds for the gradient-like system (2). To obtain the convergence result, they used an additional condition that $G, \nabla F$ satisfy an angle condition. In [15] and [16], the authors showed that the hypothesis Lojasiewicz inequality of $F$ can be extended by KurdykaLojasiewicz for convergence result. They even have the rates of convergence if $F$ satisfies Lo- 
jasiewicz inequality and $G, \nabla F$ satisfy angle and comparability condition.

In the non homogeneous case, recently $\mathrm{R}$. Chill and M. Jendoubi [7] (or Huang and Takac [14]) have shown that any bounded solution of the following gradient system

$$
u^{\prime}(t)+\nabla F(u)=f(t), t \geqslant 0,
$$

converges to a critical point of $F$ as $t$ tends to infinity if $f$ satisfies

$$
\sup _{t \in \mathbb{R}^{+}} t^{1+\mu} \int_{t}^{\infty}\|f(s)\|^{2} d s<\infty
$$

for some positive constant $\mu$. This condition shows that the forcing term $f(t)$ quickly decays to zero as $t$ goes to infinity. Their results have been generalized to some second order systems in 2], 3], 44, 9] or [10]. Moreover, M. Ghisi et. al. have estimated the decay rates for solutions of semi linear dissipative equations in [8].

Motivated by these works, we establish the convergence results for the first order nonhomogeneous gradient-like system (1) under a weaker assumption. In the other words, we will show that we can remove the strong assumption of the forcing term (3). In fact, we only need that the forcing term term $f$ belongs to $L^{1}\left(\mathbb{R}^{+}\right)$. In particular, our proof seems simpler than the proof in [7] and [14].

In this article, the convergence results will be obtained under the Kurdyka-Lojasiewicz inequality. The main difficulty comes from the generality of non-decreasing function $\Theta$ in Kurdyka-Lojasiewicz inequality. To overcome this problem, our idea is to consider $\Theta$ satisfying a subadditive property which always holds for the case of Lojasiewicz inequality. Moreover, we also establish a general abstract result for an arbitrary function which are not necessary solutions of (1). Then we apply this abstract result by replacing the energy by a suitale perturbed Lyapunov function. We believe that this general setting enables to quickly check whether convergence properties hold in specific situations.

Our article is organized as follows. In the next section, we present some notations and definitions that we use through the whole of the paper. In the last section, we also establish a general abstract result that we will apply for the main results. Then we prove the convergence of bounded solutions of the nonhomogeneous gradient-like system with the rates of convergence.

\section{Some Definitions}

In this paper, to obtain the convergence result, we assume that $G$ and $\nabla F$ satisfy the angle condition and $F$ satisfies the Kurdyka-Lojasiewicz inequality defined below.

Definition 1. We say that $G$ and $\nabla F$ satisfy the angle condition if there exists a positive number $\alpha$ such that

$$
\begin{aligned}
\langle G(u), \nabla F(u)\rangle & \geqslant \alpha\|G(u)\|\|\nabla F(u)\|, \\
\forall u & \in \mathbb{R}^{N}
\end{aligned}
$$

Using the same notation as in [13], we still denote by $Q$ the class of non-decreasing functions $\Theta \in C\left(\mathbb{R}^{+}, \mathbb{R}^{+}\right)$such that

$\Theta(0)=0, \Theta>0$ on $(0,+\infty), 1 / \Theta \in L_{l o c}^{1}\left(\mathbb{R}^{+}\right)$

Definition 2. The functions $F$ satisfies a Kurdyka-Lojasiewicz inequality at $\varphi$ if there exists $\sigma>0$ and a non-decreasing function $\Theta \in Q$ such that

$\Theta(|F(u)-F(\varphi)|) \leqslant\|\nabla F(u)\|, \quad \forall u \in B(\varphi, \sigma)$

Throughout this paper, we assume moreover that the function $\Theta$ in Kurdyka-Lojasiewicz inequality is subadditive. This means that there exists a constant $\gamma>0$ such that

$$
\Theta(x+y) \leqslant \gamma(\Theta(x)+\Theta(y)), \quad \forall x, y \in \mathbb{R}^{+}
$$

However, the Kurdyka-Lojasiewicz inequality is not sufficient to estimate the explicit convergence rate. In this case, we need a Lojasiewicz inequality.

Definition 3. We say that the function F satisfies a Lojasiewicz inequality at $\varphi$ is there exists $\beta, \sigma>0$ and $\theta \in(0,1 / 2]$ such that,

$$
\begin{gathered}
|F(u)-F(\varphi)|^{1-\theta} \leqslant \beta\|\nabla F(u)\|, \\
\forall u \in B(\varphi, \sigma)
\end{gathered}
$$

The coefficient $\theta$ is called a Lojasiewicz exponent. 
Remark 1. The Lojasiewicz inequality is a special case of Kurdyka - Lojasiewicz inequality with $\Theta(x)=(1 / \beta) x^{1-\theta}$. In particular we note that this function is subadditive by the following lemma.

Lemma 1. If $\theta \in(0,1)$ then the function $\Theta(x)=x^{1-\theta}$ is subadditive.

\section{Proof.}

For every $y \geqslant 0$ let us consider the function

$$
g_{y}(x)=(x+y)^{1-\theta}-x^{1-\theta}-y^{1-\theta}, x \geqslant 0
$$

Computing the first order derivative of $g_{y}$ at $x>$ 0 , we get

$$
g_{y}^{\prime}(x)=(1-\theta)\left((x+y)^{-\theta}-x^{-\theta}\right)
$$

Note that $x \mapsto x^{\theta}$ is non-decreasing for every $x \geqslant 0$ and $\theta \in(0,1)$, so we obtain that $g_{y}$ is nondecreasing. This implies $g_{y}(x) \leqslant g_{y}(0)=0$. The proof is complete.

\section{Convergence Results}

Let us study the main result of this paper. We first establish an abstract convergence result for arbitrary functions which are not necessary solutions of the ordinary differential equation (1). Then, we prove the convergence result under a $L^{1}$ - condition of the forcing term.

\subsection{An abstract convergence result}

Theorem 1. Let $u \in C^{1}\left(\mathbb{R}^{+}, \mathbb{R}^{N}\right)$ be bounded and $f \in L^{1}\left(\mathbb{R}^{+}, \mathbb{R}^{N}\right)$. Assume that the function $H \in C^{1}\left(\mathbb{R}^{+}\right)$is a non-increasing and $H(t)$ converges to 0 at infinity. Assume moreover that there exists a function $\Theta \in Q$ such that for every $t$ large enough, we have

$$
-\frac{H^{\prime}(t)}{\Theta(H(t))} \geqslant C\left(\left\|u^{\prime}(t)\right\|-\|f(t)\|\right),
$$

where $C$ is a positive constant. Then $u^{\prime}(t)$ belongs to $L^{1}\left(\mathbb{R}^{+}\right)$.

In particular, there exists an accumulation point $\varphi$ such that $u(t)$ converges to $\varphi$ as $t$ tends to infinity.

\section{Proof.}

Let us define $\Phi(x)=\int_{0}^{x} \frac{1}{\Theta(s)} d s, x \geqslant 0$. Since the function $H$ is non increasing and $\lim _{t \rightarrow \infty} H(t)=0$, we deduce that $\Phi$ is well-defined and $\Phi(H(t))$ converges to 0 as $t$ goes to infinity.

In the other hand, because the function $u$ is bounded, so there exists an accumulation point $\varphi$ of $u$, it means

$\varphi \in \omega[u]:=\left\{\varphi \in \mathbb{R}^{N}: \exists t_{n} \uparrow\right.$ such that $\left.u\left(t_{n}\right) \rightarrow \varphi\right\}$

From these above reasons and the hypothesis $f \in L^{1}\left(\mathbb{R}^{+}\right)$, we have that $\varphi \in \omega[u]$ and

$$
\lim _{t \rightarrow \infty} \Phi(H(t))=\lim _{t \rightarrow \infty} \int_{t}^{\infty}\|f(s)\| d s=0
$$

Hence, for every $\varepsilon>0$, we can choose $t_{0}$ large enough such that

$$
\begin{gathered}
\left\|u\left(t_{0}\right)-\varphi\right\|+C^{-1} \Phi\left(H\left(u\left(t_{0}\right)\right)\right)+ \\
+\int_{t_{0}}^{\infty}\|f(s)\| d s<\varepsilon .
\end{gathered}
$$

Let us set $t_{1}=\inf \left\{t \geqslant t_{0}:\|u(t)-\varphi\| \geqslant \varepsilon\right\}$. By (9) and continuity of the function $u$, we have $t_{1} \geqslant t_{0}$. For every $t \in\left[t_{0}, t_{1}\right]$, using the hypothesis (8), we have the estimation

$-\frac{d}{d t} \Phi(H(t))=-\frac{H^{\prime}(t)}{\Theta(H(t))} \geqslant C\left(\left\|u^{\prime}(t)\right\|-\|f(t)\|\right)$.

Integrating this estimation on $\left[t_{0}, t\right)$ for any $t \in$ $\left[t_{0}, t_{1}\right]$, we get

$$
\begin{gathered}
\int_{t_{0}}^{t_{1}}\left\|u^{\prime}(s)\right\| d s \leqslant \frac{1}{C}\left(\Phi\left(H\left(t_{0}\right)\right)-\Phi(H(t))\right) \\
+\int_{t_{0}}^{t_{1}}\|f(s)\| d s
\end{gathered}
$$

It follows from the above estimate that

$$
\begin{aligned}
& \left\|u(t)-u\left(t_{0}\right)\right\| \leqslant \int_{t_{0}}^{t_{1}}\left\|u^{\prime}(s)\right\| d s \\
& \leqslant \frac{1}{C} \Phi\left(H\left(t_{0}\right)\right)+\int_{t_{0}}^{\infty}\|f(s)\| d s
\end{aligned}
$$


We claim that $t_{1}=+\infty$. Indeed, otherwise $t_{1}<$ $+\infty$, applying the above estimate for $t=t_{1}$ and then using $(9)$, we obtain

$\left\|u\left(t_{1}\right)-\varphi\right\| \leqslant\left\|u\left(t_{1}\right)-u\left(t_{0}\right)\right\|+\left\|u\left(t_{0}\right)-\varphi\right\|<\varepsilon$

This contradicts the definition of $t_{1}$. Eventually, the estimate 10 yields that $u^{\prime}(t) \in L^{1}\left(\mathbb{R}^{+}\right)$and then we deduce $u(t)$ converges to $\varphi$ as $t$ tends to infinity by Cauchy criterion.

\subsection{Convergence under a $L^{1}-$ condition of the forcing term}

We assume that $\nabla F$ is bounded from above by a constant $K$. Let us define:

$$
V(t)=\Theta\left(K \int_{t}^{\infty}\|f(s)\| d s\right), \quad \forall t \geqslant 0
$$

We prove that the convergence result is obtained if $V \in L^{1}\left(\mathbb{R}^{+}\right)$.

Theorem 2. Let $u$ be a bounded solution of (1) and $f \in L^{1}\left(\mathbb{R}^{+}\right)$. Assume that $G, \nabla F$ satisfy the angle condition (4), $F$ satisfies the Kurdyka-Lojasiewicz inequality (5) and $\|G(u)\| \leqslant C\|\nabla F(u)\|$. If $V \in L^{1}\left(\mathbb{R}^{+}\right)$then $u(t)$ converges to as $t$ goes to infinity.

\section{Proof.}

Let us define $H(t)=F(u(t))+I(t)$, where $I(t)=\int_{t}^{\infty}\langle f(s), \nabla F(u(s))\rangle d s$ Firstly, the function $H$ is well-defined because

$$
|I(t)| \leqslant\|\nabla F\| \int_{t}^{\infty}\|f(s)\| d s .
$$

We note that since $f \in L^{1}(0,+\infty)$, so $\int_{t}^{\infty}\|f(s)\| d s \rightarrow 0$ as $t$ tends to infinity. We deduce $I(t)$ converges to 0 as $t$ goes to infinity. Next, we will prove that this function satisfies the hypotheses of Theorem 1. Indeed, using the angle condition (4), we have

$$
\begin{aligned}
H^{\prime}(t) & =\left\langle u_{t}, \nabla F(u(t))\right\rangle-\langle f(t), \nabla F(u(t))\rangle \\
& =-\langle G(u(t)), \nabla F(u(t))\rangle \\
\leqslant & -\alpha\|G(u(t))\|\|\nabla F(u(t))\| \leqslant 0 .
\end{aligned}
$$

So the function $H$ is non-increasing. Moreover, since $u$ is a bounded solution of (1), which implies that $H$ is bounded from below and there exists an accumulation point $\varphi \in \omega[u]$. Therefore, by continuity of $F$, it follows that $H(t)$ converges to $F(\varphi)$ at infinity. Without loss of generality, we may assume that $F(\varphi)=0$. In fact, we can define the energy function $H$ by $H(t)=F(u(t))-F(\varphi)+I(t)$ in general. Hence, $H(t)$ converges to 0 as $t$ goes to infinity. In the other hand, using the angle condition (4), we have

$$
\begin{gathered}
-\frac{H^{\prime}(t)}{\Theta(H(t))}=\frac{\langle G(u(t)), \nabla F(u(t))\rangle}{\Theta(H(t))} \\
\leqslant \frac{\alpha\|G(u(t))\|\|\nabla F(u(t))\|}{\Theta(H(t))} .
\end{gathered}
$$

Combining the subadditive property (6) of $\Theta$ and the Kurdyka-Lojasiewicz inequality (5), we get

$$
\begin{gathered}
\Theta(H(t)) \leqslant \gamma(\Theta(|F(u(t))|)+\Theta(|I(t)|)) \leqslant \\
\leqslant \gamma(\|\nabla F(u(t))\|+\Theta(|I(t)|)) .
\end{gathered}
$$

Moreover, we can estimate

$\frac{\|\nabla F(u(t))\|}{\|\nabla F(u(t))\|+\Theta(|I(t)|)}=1-\frac{\Theta(|I(t)|)}{\|\nabla F(u(t))\|+\Theta(|I(t)|)}$

From $(12), 13$ and $(14)$, then we obtain

$$
\begin{gathered}
-\frac{H^{\prime}(t)}{\Theta(H(t))} \geqslant \frac{\alpha}{\gamma}\|G(u(t))\| \\
-\frac{\alpha}{\gamma} \Theta(|I(t)|) \frac{\|G(u(t))\|}{\|\nabla F(u(t))\|+\Theta(|I(t)|)} \\
\geqslant \frac{\alpha}{\gamma}\|G(u(t))\|-\frac{\alpha}{\gamma} \Theta(|I(t)|) \frac{\|G(u(t))\|}{\|\nabla F(u(t))\|} \\
\geqslant \frac{\alpha}{\gamma}\|G(u(t))\|-\frac{C \alpha}{\gamma} \Theta(|I(t)|) .
\end{gathered}
$$

However, recall that $\Theta$ is non-decreasing and $\nabla F$ is bounded. So it is easy to show that $\Theta(|I(t)|) \leqslant V(t)$. Combining these estimations, we get that

$$
-H^{\prime}(t) \geqslant \frac{\alpha}{\gamma} \Theta(H(t))(\|G(u(t))\|-C V(t)) .
$$

Using the Eq. (1), we deduce that

$$
-H^{\prime}(t) \geqslant \frac{\alpha}{\gamma} \Theta(H(t))\left(\left\|u^{\prime}(t)\right\|-\|f(t)\|-C V(t)\right) .
$$


Applying Theorem 1, we finally obtain the convergence result.

Next, we will estimate the rate of convergence of bounded solutions of (1). The convergence rate will be depended on the Lojasiewicz exponent $\theta$ in (7). For more convenient, we first state the decay rate for the classical ordinary differential equation as follows:

Lemma 2. Let y be a positive solution of the following ODE:

$$
y_{t}^{\prime}+a y^{\alpha} \leqslant 0, t \geqslant 0 .
$$

If $a>0$ and $\alpha \geqslant 1$ the for $t$ large enough, we have

$$
y(t) \leqslant \begin{cases}c e^{-a t}, & \text { if } \alpha=1 \\ c t^{-1 /(\alpha-1)}, & \text { if } \alpha>1\end{cases}
$$

Proof.

In the case $\alpha=1$, we get that $y^{\prime}+a y \leqslant 0$. Writing $g(t):=e^{a t} y(t)$, we deduce that $g$ is nonincreasing. Hence, $g(t) \leqslant g(0)$, for every $t \geqslant 0$. We conclude that $y(t) \leqslant g(0) e^{-a t}$. In the second case $\alpha>1$, let us define $g(t):=(y(t))^{1-\alpha}$. This function satisfies $g^{\prime}(t) \geqslant(\alpha-1) a:=c$ which implies $g(t) \geqslant c t$ for $t$ large enough. It follows $y(t) \leqslant c t^{-1 /(\alpha-1)}$.

Theorem 3. Let $u$ be a bounded solution of (1). Assume that $\nabla F$ is bounded from below and $G, \nabla F$ satisfies the angle and comparability condition, this means there exists a constant $\nu>0$ such that

$$
\nu^{-1}\|G(u)\| \leqslant\|\nabla F(u)\| \leqslant \nu\|G(u)\|
$$

If $\sup _{t \geqslant 0} t^{1+\mu} \int_{t}^{\infty}\|f(s)\| d s<\infty$ for some constant $\mu>0$ and $F$ satisfies Lojasiewicz inequality with Lojasiewicz exponent $\theta \in\left(0, \frac{\mu}{1+\mu}\right)$ then $u(t)$ converges to $\varphi$ as $t$ goes to infinity. We even have the convergence rate as follows:

$$
\begin{aligned}
\|u(t)-\varphi\| & \leqslant O\left(t^{-(\mu-\theta-\theta \mu)}\right) \\
& + \begin{cases}c e^{-a \nu t}, & \text { if } \theta=1 / 2 \\
c t^{-\theta /(1-2 \theta)}, & \text { if } \theta \in(0,1 / 2)\end{cases}
\end{aligned}
$$

\section{Proof.}

We have $\sup _{t \geqslant 0} t^{1+\mu} \int_{t}^{\infty}\|f(s)\| d s<\infty$ and now we deduce that $\int_{t}^{\infty}\|f(s)\| d s \leqslant C t^{-(1+\mu)}$. Therefore,

$$
V(t) \leqslant \Theta\left(C K t^{-(1+\mu)}\right)=C_{1} t^{-(1+\mu)(1-\theta)} .
$$

For every $\theta \in\left(0, \frac{\mu}{1+\mu}\right)$, we have $(1+\mu)(1-\theta)>$ 1. It turns out that $V \in L^{1}\left(\mathbb{R}^{+}\right)$. Applying Theorem 2, we have the convergence result of bounded solution $u$ and we even obtain the following estimation

$$
\|u(t)-\varphi\| \leqslant-\frac{\gamma}{\alpha} \frac{d}{d t} \Phi(H(t))+\|f(t)\|+C V(t) .
$$

Integrating this equality on $[t, \infty)$, we get that:

$$
\begin{gathered}
\|u(t)-\varphi\| \leqslant \int_{t}^{\infty}\left\|u^{\prime}(s)\right\| d s \\
\leqslant \int_{t}^{\infty}\|f(s)\| d s+C \int_{t}^{\infty} V(s) d s+\frac{\gamma}{\alpha} \Phi(H(t)) \\
\leqslant O\left(t^{-(1+\mu)}\right)+O\left(t^{-(\mu-\theta-\theta \mu)}\right)+\frac{\gamma}{\alpha} \Phi(H(t)) \\
\leqslant O\left(t^{-(\mu-\theta-\theta \mu)}\right)+\frac{\gamma}{\alpha} \Phi(H(t)) .
\end{gathered}
$$

In the other hand, by using the angle and comparability condition (15) and the Lojasiewicz inequality, we deduce that

$$
-\frac{d}{d t} \Phi(H(t)) \geqslant \nu \frac{\|\nabla F(u(t))\|^{2}}{\Theta(H(t))} \geqslant \nu \Theta(H(t)) .
$$

Recall that in the Remark 1 if $F$ satisfies Lojasiewicz inequality then $\Theta(x)=(1 / \beta) x^{1-\theta}$ and $\Phi(x)=(\beta / \theta) x^{\theta}$ It follows that $\Theta(x)=$ $a \Phi(x)^{\frac{1-\theta}{\theta}}$, where $a=a(\theta, \beta)$ is a positive constant. Then we deduce from (17) as follows

$$
\frac{d}{d t} \Phi(H(t))+a \nu \Phi(H(t))^{\frac{1-\theta}{\theta}} \leqslant 0
$$

Applying Lemma 2 for the above ordinary differential equation, we get that

$$
\Phi(H(t)) \leqslant\left\{\begin{array}{lc}
c e^{-a \nu t}, & \text { if } \theta=1 / 2, \\
c t^{-\theta /(1-2 \theta)}, & \text { if } \theta \in(0,1 / 2) .
\end{array}\right.
$$

Combining two above estimations, we obtain the convergence rate (16). The proof is complete. 


\section{Conclusion}

In this article, we establish some convergence results of bounded solutions for non homogeneous first order gradient-like system under $L^{1}$ condition of forcing term. We also provide an estimation of convergence rate. The asymptotic behavior of solutions for general second order gradient-like system is still interesting for many people. We hope to study this problem in future works.

\section{References}

[1] ABSIL, P.-A. and K. KURDYKA. On the stable equilibrium points of gradient systems. Systems Control Lett. 2006, vol. 55, iss. 7 , pp. $573-577$.

[2] BARTA, T. Convergence to equilibrium of relatively compact solutions to evolution equations. Electron. J. Differential Equations. 2014, iss. 81.

[3] BEGOUT, P., J. BOLTE and M. A. JENDOUBI. On damped second-order gradient systems. J. Differential Equations. 2015, vol. 259 , iss. 7 , pp. $3115-3143$.

[4] CABOT, A., P. FRANKEL. Asymptotics for some semilinear hyperbolic equations with non-autonomous damping. J. Differential Equations. 2012, vol. 252, iss. 1, pp. 294-322.

[5] CHILL, R. On the łojasiewicz-Simon gradient inequality. J. Funct. Anal. 2003, vol. 201, iss. 2, pp. 572-601.

[6] CHILL, R., A. HARAUX and M. A. JENDOUBI. Applications of the łojasiewiczSimon gradient inequality to gradient-like evolution equations. Anal. Appl. (Singap.). 2009 , vol. 7 , iss. 4 , pp. $351-372$.

[7] CHILL, R. and M. A. JENDOUBI. Convergence to steady states in asymptotically autonomous semilinear evolution equations. Nonlinear Anal. 2003, vol. 53, iss. 7-8, pp. 1017-1039.
[8] GHISI, M., M. GOBBINO and A. HARAUX. A description of all possible decay rates for solutions of some semilinear parabolic equations. J. Math. Pures Appl. 2015, vol. 103 iss. 4, pp. 868-899.

[9] GRASSELLI, M. and M. PIERRE. Convergence to equilibrium of solutions of the backward Euler scheme for asymptotically autonomous second-order gradient-like systems. Commun. Pure Appl. Anal. 2012, vol. 11, iss. 6, pp. 2393-2416.

[10] HARAUX, A. and M. A. JENDOUBI. Convergence of solutions of second-order gradient-like systems with analytic nonlinearities. J. Differential Equations. 1998, vol. 144 iss. 2, pp. 313-320.

[11] HARAUX, A. and M. A. JENDOUBI. On the convergence of global and bounded solutions of some evolution equations. J. Evol. Equ.. 2007, vol. 7, no. 3, pp. 449-470.

[12] HARAUX, A. and M. A. JENDOUBI. The convergence problem for dissipative autonomous systems. SpringerBriefs in Mathematics. Springer, Cham; BCAM Basque Center for Applied Mathematics Bilbao. 2015.

[13] HUANG, S.-Z. Gradient inequalities. Volume 126 of Mathematical Surveys and Monographs American Mathematical Society. 2006.

[14] HUANG, S.-Z. and P. TAKAC. Convergence in gradient-like systems which are asymptotically autonomous and analytic. Nonlinear Anal. 2001, vol. 46, iss. 5, Ser. A: Theory Methods, pp. 675-698.

[15] MERLET, B. and T.-N. NGUYEN. Convergence to equilibrium for discretizations of gradient-like flows on Riemannian manifolds. Differential Integral Equations. 2013, vol. 26, iss. 5-6, pp. 571-602.

[16] NGUYEN, T.-N. Convergence to equilibrium for discrete gradient-like flows and An accurate method for the motion of suspended particles in a Stokes fluid. Thesis (Ph.D.)-Ecole Polytechnique. France, 2013. 
[17] SIMON, L. Asymptotics for a class of nonlinear evolution equations, with applications to geometric problems. Ann. of Math. (2). 1983, vol. 118, iss. 3, pp. 525-571.

\section{About Authors}

Minh-Phuong TRAN was born in Ho Chi Minh city, Vietnam, in 1986. She received B.Sc. in Mathematics from the HCMC University of Education, Vietnam, 2008, and the M.Sc. and Ph.D. degrees in Applied Mathematics from the University of Orléans, Orléans, France, in 2009 and 2012, respectively. In 2013, she joined the Department of Mathematics and Applications in Ecole Normale Supérieur, Paris, France, as a Postdoctoral Researcher. And in 2014, she became a Lecturer in Faculty of Mathematics-
Statistics in Ton-Duc-Thang university, HCMC, Vietnam. Her current research interests include Numerical Analysis, Image Procesing, Inverse Problem in Imaging, Computational Fluid Dynamics, Ordinary and Partial Differential Equations.

Thanh-Nhan NGUYEN was born in Ben Tre, Vietnam, in 1986. He received M.Sc. degree in Applied Mathematics from the University of Orléans, Orléans, France, in 2010 and Ph.D. degree in Applied Mathematics from École Polytechnique, Paris, France, in 2013. He is working in Department of Mathematics, Ho Chi Minh City University of Education, HCMC, Vietnam, as a Lecturer. His current research interests include Ordinary and Partial Differential Equations, Numerical Analysis, and Computational Fluid Dynamics.

"This is an Open Access article distributed under the terms of the Creative Commons Attribution License, which permits unrestricted use, distribution, and reproduction in any medium, provided the original work is properly cited (CC BY 4.0)." 